The accumulation of boundary doubling for modified tent maps

Glendinning, Paul

2003

MIMS EPrint: 2006.100

Manchester Institute for Mathematical Sciences

School of Mathematics

The University of Manchester

\footnotetext{
Reports available from: http://eprints.maths.manchester.ac.uk/

And by contacting: The MIMS Secretary

School of Mathematics

The University of Manchester

Manchester, M13 9PL, UK
} 


\title{
The accumulation of boundary doubling for modified tent maps
}

\author{
Paul Glendinning
}

\begin{abstract}
We describe the transition to chaos via boundary doubling for a particularly simple class of map. In this two parameter family of maps the accumulation of boundary doubling occurs on a curve in parameter space. We characterize this curve and use relate the form of this curve to a novel set of difference equations with proportional delay.
\end{abstract}

\section{Introduction}

The transition to chaos in the sense of positive topological entropy is one of the fundamental problems of applied dynamical systems. In [5] (see also [7]) we showed that if $f_{\mu}: I \rightarrow \mathbf{R}$ is a family of unimodal, or one hump, maps of the interval such that the image of the critical point of the map lies outside the interval for all relevant values of the parameter $\mu$, then the transition to chaos can be via an infinite sequence of boundary bifurcations. At the boundary bifurcation, the boundary of the interval is periodic, creating periodic orbit of period $2^{n}$. If these bifurcations occur at parameter values $\mu_{n}$, then under some fairly weak assumptions about the maps being considered

$$
\left|\mu_{n+1}-\mu_{n}\right| \rightarrow C\left|\mu_{n}-\mu_{n-1}\right|^{2}
$$

as $n \rightarrow \infty$ where the constant $C$ depends on the family of maps being considered. It was also pointed out that such maps might find application in models with some catastrophic breakdown threshold above which the model ceases to be a good description of the phenomenon. The simplest examples of these bifurcations arises in modified tent maps, and it is these which we consider in more detail below.

A tent map with threshold is a standard tent map $[3,8]$ with the added restriction that the map is undefined if $x>\mu$ for some $\mu \in(0,1)$, i.e.

$$
T_{\mu, s}(x)= \begin{cases}s x & \text { if } 0 \leq x \leq s^{-1} \mu \\ \text { undefined } & \text { if } s^{-1} \mu<x<s^{-1}(s-\mu) \\ s(1-x) & \text { if } s^{-1}(s-\mu) \leq x \leq \mu\end{cases}
$$

with $s>1$ and $0<\mu \leq 1$. It is these maps which will be the focus of our attention in the remainder of this paper. Note that the boundary bifurcations will occur 
through the right hand end point of the interval $[0, \mu]$, i.e. the point $x=\mu$ will become periodic.

The maps $T_{\mu, s}$ with the slight modification that $T_{\mu, s}(x)=\mu$ on the central interval, which makes the map a continuous map of the interval into itself, were considered briefly by Derrida, Gervois and Pomeau [4] in the context of perioddoubling, and the renormalization argument of the next section can be found there. I suspect that little of what is contained here, except perhaps the last section, would be new to them. The special case of $s=2$ is considered in [9] and related results can be found in [1].

\section{Renormalization}

If $\mu<s^{-1}(s-\mu)$ then the only recurrent dynamics in $[0,1]$ is the fixed point at $x=0$. As $\mu$ increases through $\mu_{0}=s(s+1)^{-1}$ a new fixed point, $x_{0}$ is created, and this fixed point has a unique preimage $y_{0}$ in $x<s^{-1} \mu$. An easy calculation yields

$$
x_{0}=s(s+1)^{-1}, \quad y_{0}=(s+1)^{-1}
$$

If $\mu>\mu_{0}$ then we can consider the second iterate, $T_{1}$, of the map on the two intervals in $\left[y_{0}, x_{0}\right]$ on which it is well defined, and these branches of the second iterate map the intervals they are defined on into $\left[y_{0}, x_{0}\right]$ provided $\mu<\tilde{\mu}_{1}$ where

$$
\tilde{\mu}_{1}=\frac{s^{2}+s-1}{s(s+1)} .
$$

After an affine, orientation reversing change of variable so that the induced map is now defined on the interval $[0,1]$ it is easy to show that if $\mu \in\left(\mu_{0}, \tilde{\mu}_{1}\right]$ then the induced map $T_{1}$ is again a map of the form (2), $T_{M, S}$, where

$$
M=\frac{s-s(s+1)(1-\mu)}{s-1} \quad \text { and } \quad S=s^{2}
$$

Note that if $\lim _{\mu \downarrow \mu_{0}}$ then $M=0$ and if $\mu=\tilde{\mu}_{1}$ then $M=1$ so the entire range of dynamics available to $T_{M, S}$ is realised by the induced map $T_{1}$. In particular, there is $\mu_{1}$ at which an orbit of period one for the induced map (period two for the original map) is created by boundary bifurcation, and a new induced map $T_{2}$ (the second iterate of the second iterate of $\left.T_{\mu, s}\right)$ can be defined on $\left(\mu_{1}, \tilde{\mu}_{2}\right]$, where $\tilde{\mu}_{2}$ is the analogous parameter value to $\tilde{\mu}_{1}$.

We now use the standard bootstrap argument as is used in the case of perioddoubling cascades. Set $\tilde{\mu}_{0}=1$. Then proceeding inductively we see that if $\mu \in$ $\left[\mu_{n}, \tilde{\mu}_{n}\right]$ then the induced map $T_{n}$ (made up of parts of the $2^{n}$ th iterate of $T$ ) is well defined on an interval which has a point $x_{n-1}$ of period $2^{n-1}$ at one end point. If $\mu=\mu_{n}$, an orbit of period $2^{n}$ is created by boundary bifurcation, and if $\mu=\tilde{\mu}_{n}$ the two branches of the induced map stretch over the interval on which the map is defined and so, by standard arguments [8], the topological entropy of 
the original map is $2^{-n} \log 2$. The intervals $I_{n}=\left[\mu_{n}, \tilde{\mu}_{n}\right]$ form a nested sequence of closed intervals, and so

$$
\mu_{\infty}=\bigcap_{n=0}^{\infty} I_{n}
$$

is non-empty, and is the accumulation point of the two sequences, $\left(\mu_{n}\right)$ and $\left(\tilde{\mu}_{n}\right)$ (strictly speaking we have not shown that $\mu_{\infty}$ is a singleton). If $\mu=\mu_{\infty}$ then the renormalization process (the process of defining induced maps) can be repeated infinitely often. If $\mu<\mu_{\infty}$ then the only periodic orbits of $T_{\mu, s}$ have periods $2^{n}$, $n=0,1, \ldots, N$, for some finite $N$ and so the topological entropy of the map is zero, whilst if $\mu>\mu_{\infty}$, then $\mu>\tilde{\mu}_{m}$ for some $m$ and so the entropy of the map is greater than or equal to $2^{-m} \log 2$.

\section{Scaling}

Equation (5) makes it relatively straightforward to verify the scaling, (1). We have already shown that $\mu_{0}=s(s+1)^{-1}$. If $T$ has parameter $\mu=\mu_{n}$, with $\mu_{n}$ defined in section 2 , then the induced map $T_{n}$ has parameters $\left(M_{n}, S_{n}\right)$ with $M_{n}=S_{n}\left(S_{n}+1\right)^{-1}$. Similarly, if $T$ has $\mu=\mu_{n+1}$ then $T_{n+1}$ has parameters $\left(M_{n+1}, S_{n+1}\right)$ with $S_{n+1}=S_{n}^{2}$ by $(5)$ and $M_{n+1}=S_{n+1}\left(S_{n+1}+1\right)^{-1}$. Setting $\mu=M_{n}, s=S_{n}$ and $M=M_{n+1}$ in the first equation of (5) we find

$$
M_{n}=\frac{S_{n}^{2}+\left(S_{n}-1\right) M_{n+1}}{S_{n}\left(S_{n}+1\right)}
$$

where we think of $M_{n+1}$ as a function of $S_{n}^{2}$.

Now, at the parameter values $\mu_{n}, \mu_{n+1}$ and $\mu_{n+2}, T_{n}$ is well defined and the corresponding parameters for this induced map are $M_{n, 0}, M_{n, 1}$ and $M_{n, 2}$ where

$$
M_{n, 0}=\frac{S_{n}}{S_{n}+1}, M_{n, 1}=\frac{S_{n}^{2}}{S_{n}^{2}+1}, \text { and } M_{n, 2}=\frac{S_{n}\left(S_{n}^{3}-S_{n}+1\right)}{S_{n}^{4}+1}
$$

as is verified using the expression already given for $\mu_{0}$ and two applications of (7). Now let

$$
\Delta_{n, m}=M_{n, m+1}-M_{n, m}
$$

and think of $\Delta_{n, m}$ as a function of $S_{n}=S_{n-1}^{2}$, cf. (11). Defining $T_{0}$ to be the original map (2) we see that equation (1) is equivalent to

$$
\Delta_{0, n} \rightarrow C \Delta_{0, n-1}^{2} \text { as } n \rightarrow \infty
$$

and it is this which we wish to demonstrate now.

If $T_{n}$ has a boundary bifurcation which creates an orbit of period $2^{m}$ at $M_{n, m}$ then $T_{n-1}$ has a boundary bifurcation creating an orbit of period $2^{m+1}$ at $M_{n-1, m+1}$ which can be obtained from $M_{n, m}$ using (7). Hence

$$
\Delta_{n-1, m+1}=\frac{S_{n-1}-1}{S_{n-1}\left(S_{n-1}+1\right)} \Delta_{n, m}\left(S_{n-1}^{2}\right)
$$


In other words, if $n$ is large, so $S_{n}$ is large, and $\Delta_{n, m} \sim S_{n}^{-r_{n, m}}$ then $\Delta_{n-1, m+1} \sim$ $S_{n-1}^{-r_{n-1, m+1}}$ where $r_{n-1, m+1}=2 r_{n, m}+1$, i.e.

$$
r_{n-k, n+k}=2^{k} A-1, \quad \text { with } A=r_{n, m}+1 \text {. }
$$

for $k=0, \ldots, n$. A simple calculation using (8) shows that

$$
\Delta_{n, 1}=\frac{S_{n}\left(S_{n}-1\right)}{\left(S_{n}^{2}+1\right)\left(S_{n}+1\right)} \quad \Delta_{n, 2}=\frac{S_{n}\left(S_{n}-1\right)^{2}}{\left(S_{n}^{4}+1\right)\left(S_{n}^{2}+1\right)}
$$

so $r_{n, 1}=1$ and $r_{n, 2}=3$, and hence $r_{0, n+1}=2.2^{n}-1$ and $r_{0, n+2}=4.2^{n}-1$. Equation (10) now follows since

$$
\Delta_{0, n+1}^{2} \sim s^{-4.2^{n}+1} \text { and } \Delta_{0, n+1}^{2} \sim s^{-4.2^{n}+2}=s . s^{-4.2^{n}+1}
$$

Note that a similar argument gives the same scaling result for the parameters $\tilde{\mu}_{n}$ and this, together with the remarks on entropy at the end of section two show that the entropy increases like the reciprocal of the logarithm of $\left|\tilde{\mu}_{n}-\mu_{\infty}\right|$, a result known to Derrida et al [4], see also [5, 9].

\section{The accumulation curve}

The accumulation of boundary doublings occurs at a parameter $\mu_{\infty}$ which is a function of $s$. The locus of this accumulation in the full two parameter space $(\mu, s)$ will be discussed here. On this locus, the map $T_{0}$ can be renormalized infinitely many times, and hence so can the induced map $T_{1}$. Hence, if the locus takes the form $\mu=F(s)$ then from (5) we find that

$$
(s-1) F\left(s^{2}\right)=s-s(s+1)(1-F(s))
$$

with $s>0$. Note that $\lim _{s \rightarrow 1} F(s)=\frac{1}{2}$ and $\lim _{s \rightarrow \infty} F(s)=1$ as we would expect. It is easier to approach (15) using the inverse of $s$, so if $G\left(s^{-1}\right)=F(s)$ and $t=s^{-1}$ then (15) may be rewritten as

$$
G(t)=\frac{1}{1+t}+\frac{t(1-t)}{1+t} G\left(t^{2}\right)
$$

Replacing $t$ by $t^{2}$ throught to obtain an expression for $G\left(t^{2}\right)$ in terms of $G\left(t^{4}\right)$ and so

$$
G(t)=\frac{1}{1+t}+\frac{t(1-t)}{(1+t)\left(1+t^{2}\right)}+\frac{t^{3}(1-t)\left(1-t^{2}\right)}{(1+t)\left(1+t^{2}\right)} G\left(t^{4}\right) .
$$

It is now relatively straightforward to repeat this process, giving

$$
G(t)=\sum_{n=0}^{\infty} a_{n}(t) t^{2^{n}-1}
$$

where

$$
a_{0}=\frac{1}{1+t}, \quad a_{1}=\frac{1-t}{(1+t)\left(1+t^{2}\right)}, \quad a_{n}=\frac{(1-t) \prod_{r=0}^{n-2}\left(1-t^{2^{r}}\right)}{\left(1+t^{2^{n-1}}\right)\left(1+t^{2^{n}}\right)}, \quad n \geq 2 .
$$


An alternative approach to the solution of (16) is to pose a formal power series solution $G(t)=\sum_{0}^{\infty} y_{n} t^{n}$. After rewriting (16) as $(1+t) G(t)=1+t(1-t) G\left(t^{2}\right)$, substitution of this formal power series solution gives a linear differnece equation with proportional delay for the coefficients:

$$
y_{2 n+1}=-y_{2 n}+y_{n}, \quad y_{2 n+2}=-y_{2 n+1}-y_{n}
$$

with the initial condition $y_{0}=1$. These equations differ to those studied by Buhmann and Iserles $[2,6]$ for a discretized model of the pantograph equation on the boundary of stability of the trivial solution only in the sign of $y_{n}$ in the second equation. Equations (20) have a curious self-similarity property. If $z_{k}=y_{k+1}+y_{k}$ then $z_{2 n+1}=-z_{2 n}$, so we need only consider the odd or the even $z_{k}$. Consider the even case and let $z_{2 k}=b_{k}$. Then, noting that $z_{2 n+2}+z_{2 n}=z_{n}$ we find that the difference equation for $b_{k}$ is precisely (20) - even the initial condition is the same! I have been unable to find a simple interpretation of this result, but the coefficients of the solutions to the discretized pantograph equations also have a fractal structure [6].

\section{References}

[1] K.M. Brucks, M. Misiurewicz and C. Tresser, Monotonicity properties of the family of trapezoidal maps, Comm. Math. Phys. 137 (1991), 1-12.

[2] M.D. Buhmann and A. Iserles, On the dynamics of a discretized neutral equation, IMA J. Num. Anal., 12 (1992), 339-363.

[3] P. Collet and J.P. Eckmann, Iterated maps on the interval as dynamical systems, Birkhauser, Boston, 1980.

[4] B. Derrida, A. Gervois and Y. Pomeau, Universal metric properties of bifurcations of endomorphisms, J. Phys. A, 12 (1978), 269-296.

[5] P. Glendinning, Transitions to chaos in unimodal maps of the interval to the real line, UMIST Appl. Math. Rep. preprint 00/2 (2000).

[6] A. Iserles, Exact and discretized stability of the pantograph equation, Appl. Numer. Math., 24 (1997), 295-308.

[7] H. Kokubu, M. Komuro and H. Oka, Multiple homoclinic bifurcations from orbit-flip. I. Successive homoclinic doublings, Int. J. Bif. \& Chaos, 6 (1996), 833-850.

[8] S. van Strien, Smooth dynamics on the interval, New Directions in Dynamical Systems, ed. T. Bedford and J. Swift, Cambridge University Press, Cambidge, 1988.

[9] K. Zyczkowski and E.M. Bollt, On the entropy devil's staircase in a family of gap-tent maps, Physica D, 132 (1999), 392-410.

Department of Mathematics,

UMIST,

P.O. Box 88,

Manchester M60 1QD, U.K.

E-mail address: p.a.glendinning@umist.ac.uk 